\title{
Contrast Enhanced Magnetic Resonance Angiography with Gadofosveset Used in Detection and Localization of Bleeding
}

\author{
D. Durant, G. Annamalai \\ Sunnybrook Health Sciences Centre, Toronto, Canada \\ Email: dean.durant@gmail.com
}

Received 19 November 2013; revised 19 December 2013; accepted 26 December 2013

Copyright (C) 2014 by authors and Scientific Research Publishing Inc.

This work is licensed under the Creative Commons Attribution International License (CC BY).

http://creativecommons.org/licenses/by/4.0/

(c) (i) Open Access

\begin{abstract}
It has been established that internal hemorrhage can be detected and localized with noninvasive and minimally invasive imaging methods such as CT angiography, conventional angiography and nuclear scintigraphy. The use of steady-state MRI with the relatively new intravascular contrast agent gadofosveset offers the possibility of accurate anatomic localization of bleeding without the use of ionizing radiation or iodinated contrast media. To illustrate this assertion, we describe two cases from our institution in which MR angiography was successfully performed specifically for this indication.
\end{abstract}

\section{Keywords}

MRI; Hemorrhage; Gadofosveset

\section{Introduction}

Clinical hemorrhage can be broadly categorized into traumatic and non-traumatic etiologies. In the non-traumatic category gastrointestinal bleeding is common, accounting for over $20 \%$ of emergency room admissions [1]. External bleeding such as due to trauma is usually easily localized and managed.

In cases of internal bleeding, the clinician relies on accurate and thorough history and physical examination in order to ascertain the site and possible cause of hemorrhage. While there should be no substitute for thorough clinical assessment, in recent years there has been a strong drive towards developing non-invasive methods of detecting, localizing and treating hemorrhage. For purposes of this article, we focus on the detection of gastrointestinal and bronchial artery bleeding, utilizing two cases to highlight a novel non-invasive method of localizing hemorrhage using magnetic resonance imaging (MRI) with a blood-pool contrast agent. 


\section{Materials and Methods}

Retrospective analysis of two consecutive cases in which Magnetic Resonance Angiography (MRA) was performed using gadofosveset with localization of bleeding as the indication. Patient records and imaging were reviewed.

Permission for chart review was obtained from the Health Data Resources Department.

Case 1: 70 y/o female presented to the ER department with a 2-month history of melena stools and worsening weakness, which led to suspicion of upper gastrointestinal (GI) bleeding. On the day of her admission to hospital she experienced an episode of passing bright red blood per-rectum. Of note, she had been on long-term anticoagulation for a mechanical mitral valve. Upper GI endoscopy demonstrated fundal gastric polyps and no signs of bleeding. Colonoscopy demonstrated scattered blood throughout the colon but the source of hemorrhage was not identified. Abdominal MRA with steady-state imaging was then performed and showed increasing T1 signal in the ascending colon on sequential images, with distal propagation on delayed images due to peristalsis causing movement of contrast (Figure 1). Conventional digital subtraction angiography (DSA) was performed the following day due to further hemoglobin decrease and continued melena. Faint linear blush was seen along the antimesenteric border of the ascending colon arising from a distal ileocolic arterial branch (Figure 2). Due to vascular tortuosity and small caliber, it was not possible to get subselective enough to allow embolization.

Non-contrast CT immediately after DSA demonstrated high-density intraluminal contrast in the cecum and right colon confirming extravasation (Figure 3). Repeat colonoscopy two days later showed no active bleeding but multiple ulcers and erosions in the ascending colon, reported as possible sources of bleeding (Figure 4). Over the subsequent few days her hemoglobin stabilized with no further episodes of bleeding and she was discharged 12 days following admission.

Case 2: 50 y/o female patient transferred from an outside institution presented with recurrent large-volume hemoptysis and a complex medical history including pneumonia, aspergillosis, previous lung reduction surgery and bronchial artery embolization, which was performed at an outside institution. An MRA was performed on admission, immediately followed by DSA. MRA demonstrated an anomalous dilated vessel arising from the right thyrocervical trunk to supply a portion of abnormal right upper lobe (Figure 5), which contained multiple cystic spaces and diffuse fibrosis. High T1 signal seen on the Magnetic Resonance Direct Thrombus Imaging (MRDTI) sequence [2] is indicative of recent hemorrhage into some of the cystic spaces in the right lung (Figure 6). DSA was guided by MRA findings. The abnormal branch of the right thyrocervical trunk was selected (Figures 7(a) and (b)) and seen to supply a portion of the previously embolized bronchial artery via collaterals. No active extravasation was demonstrated, however the abnormal vessels were empirically embolized using microspheres. The patient suffered no further hemoptysis and was discharged.

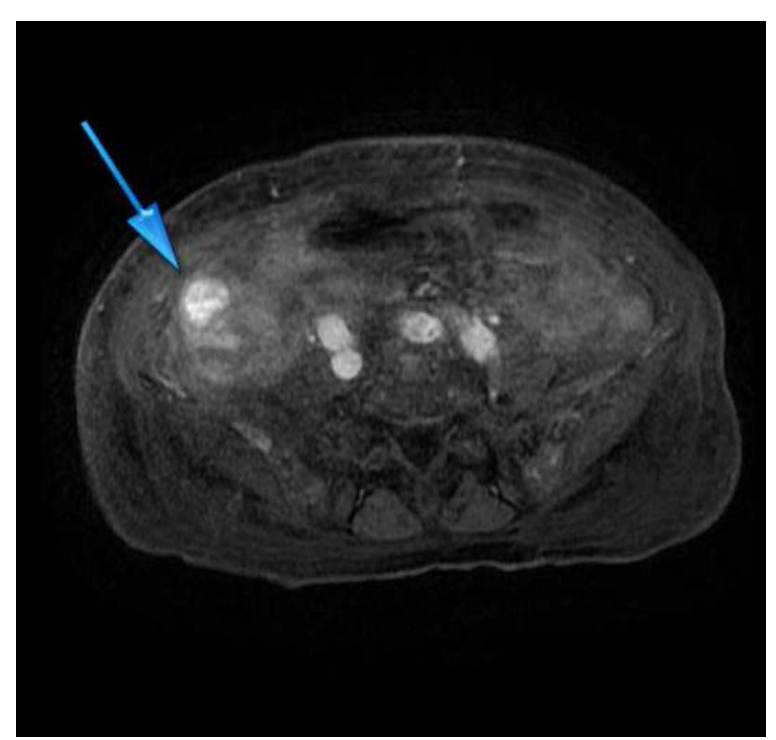

Figure 1. Axial steady-state T1 weighted MR image. High signal within ascending colon in keeping with leaking contrast material. 


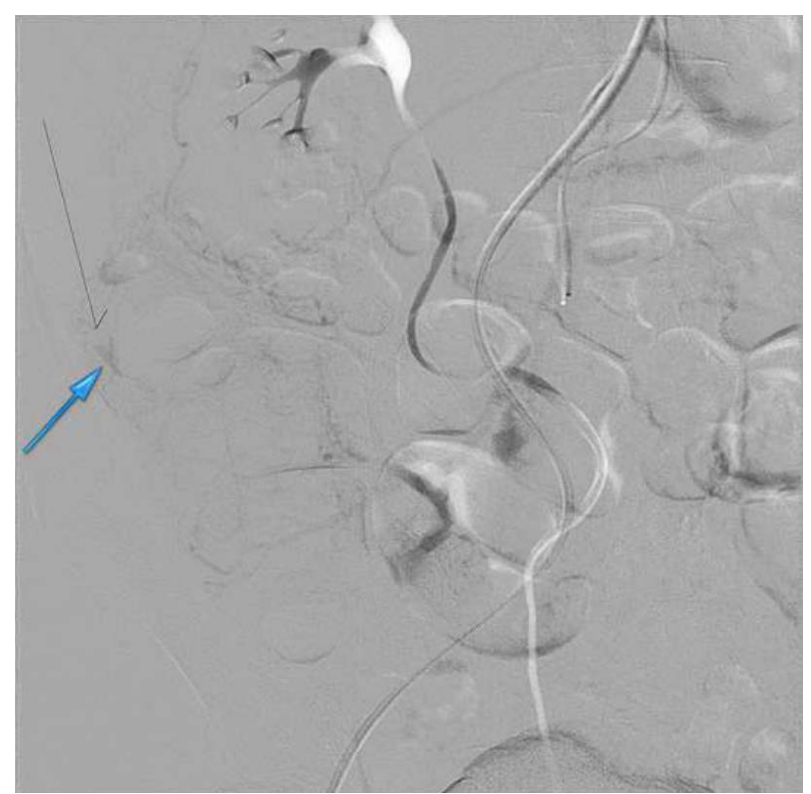

Figure 2. Linear blush seen from bleeding ileocolic branch during mesenteric angiography performed the day after MR angiogram.

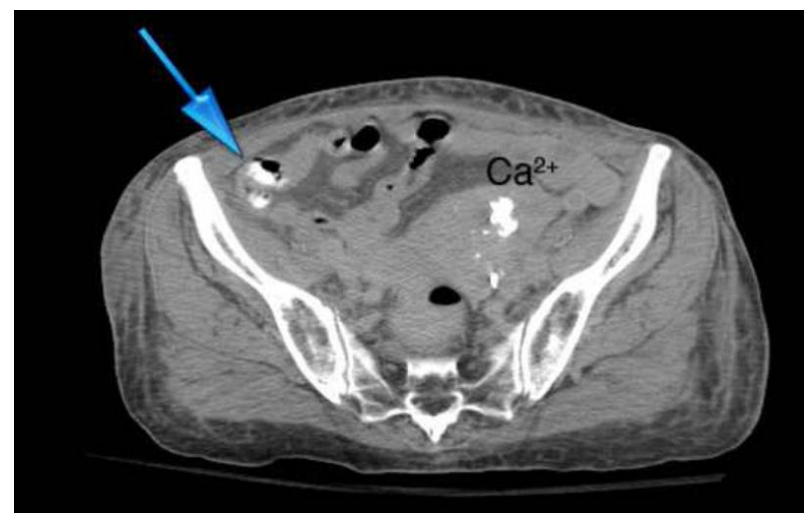

Figure 3. Non-contrast CT scan performed immediately after conventional angiogram. High density contrast seen in ascending colon, extravasated during angiogram.

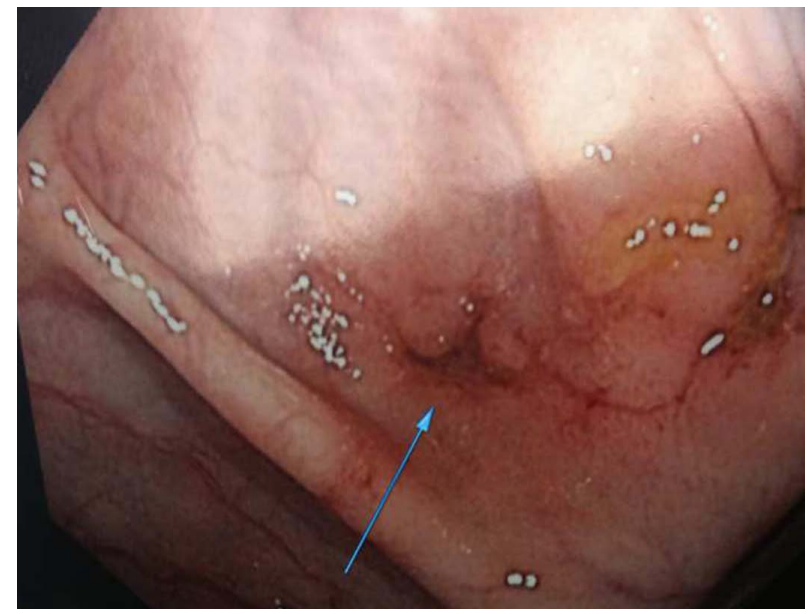

Figure 4. Image from colonoscopy showing one of the ascending colonic ulcers identified. 


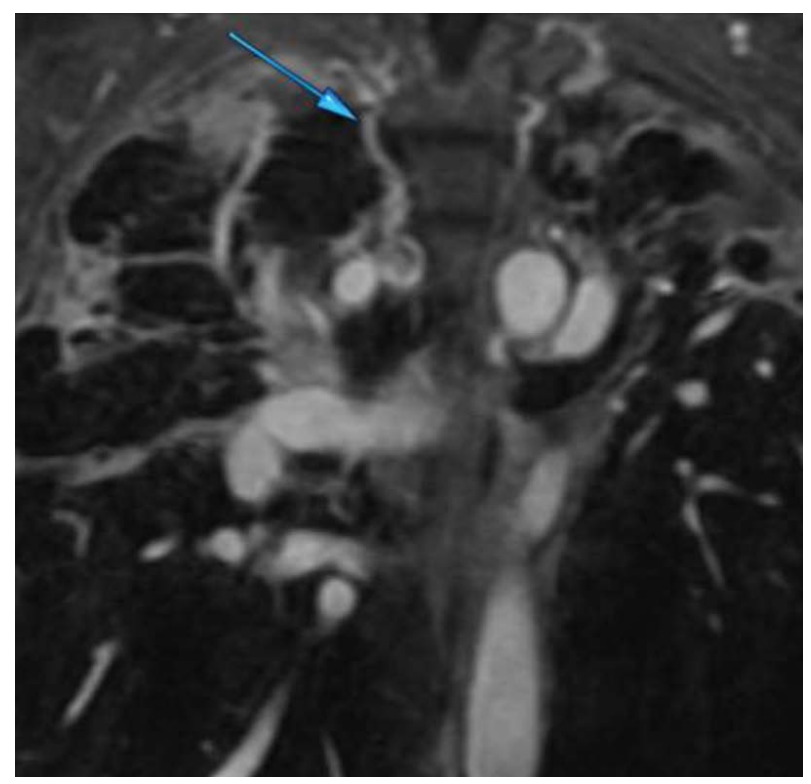

Figure 5. Coronal image from Steady-State T1 Weighted MR Series. Dilated anomalous bronchial artery fed by collaterals from right thyrocervical trunk, which in turn is also enlarged.

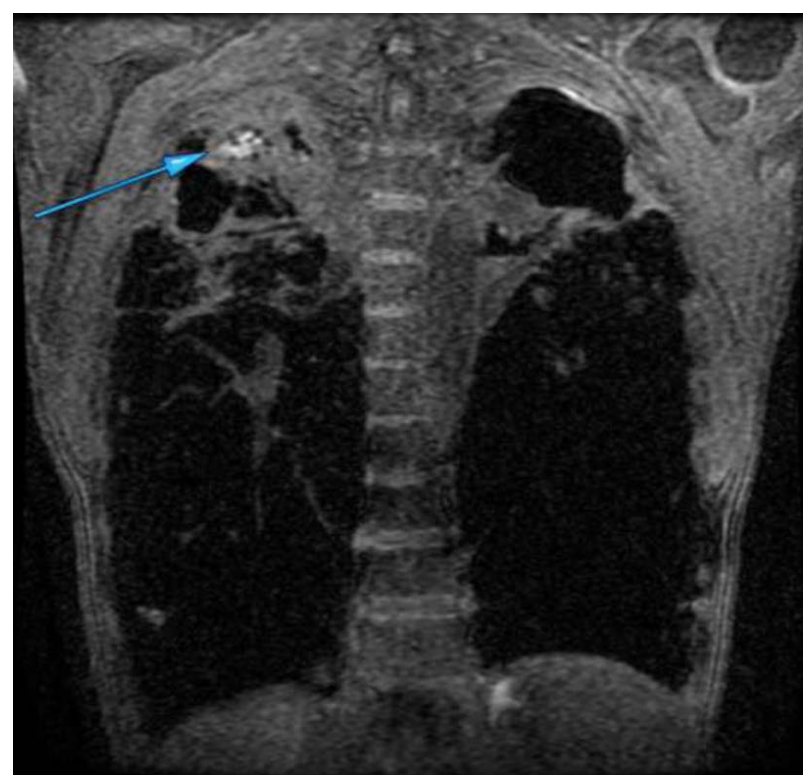

Figure 6. MRDTI image showing localized high signal at the right lung apex in keeping with acute blood products.

Technique: MRI: 1.5T GE Signa Twinspeed scanner.

MRDTI: TR 4.4ms, TE $1.7 \mathrm{~ms}$ and flip angle 15 degrees. This sequence is optimized for detection of methemoglobin and therefore subacute thrombosis.

Steady-state: Images acquired at intervals (immediate, 30 seconds, 1 minute, 3 minutes, 5 minutes and 10 minutes) after intravenous injection of 10 cc gadofosveset, TR 3.9 ms, TE 1.4 ms and flip angle 35 degrees.

DSA: Phillips Allura Xper FD20 fluoroscopy machine. CT: GE 64 slice Lightspeed VCT.

\section{Results}

In the first case, MRA demonstrated increasing signal in the ascending colon, accurately localizing the site of slow hemorrhage and guiding the subsequent DSA. 


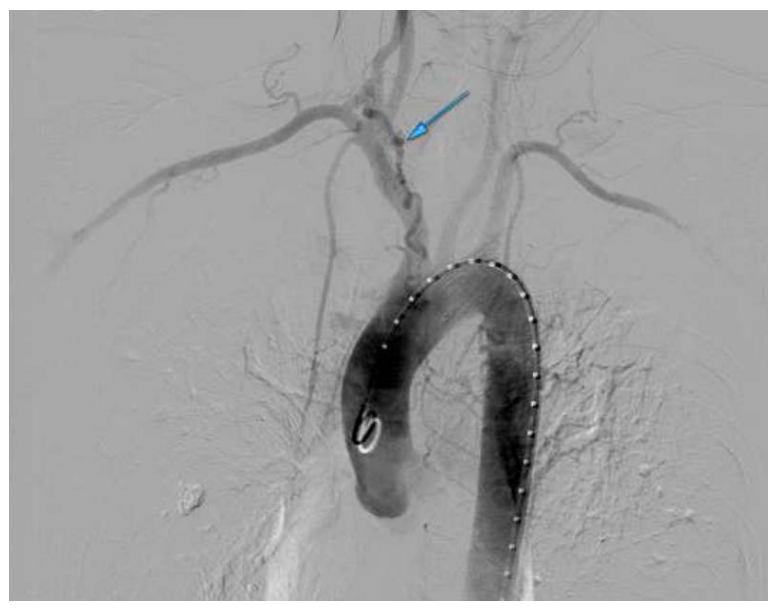

(a)

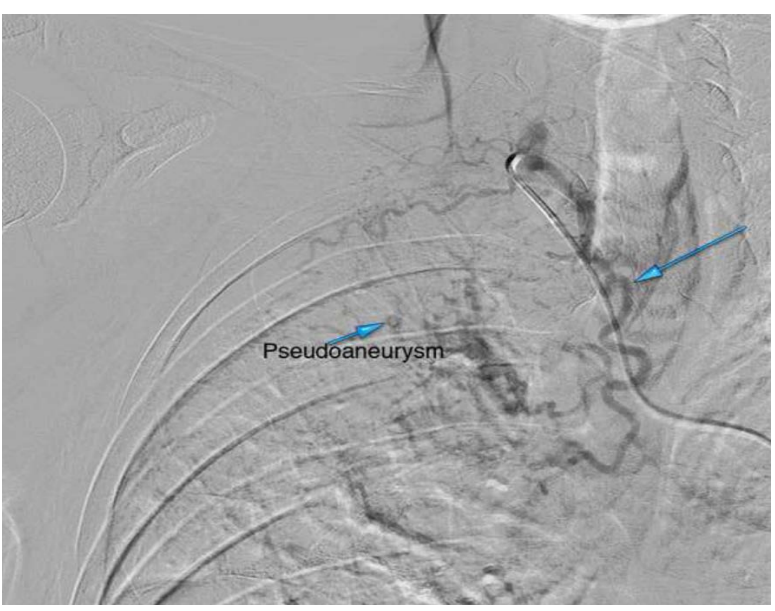

(b)

Figure 7. (a), (b) Arch aortogram (a) highlighted by dilated thyrocervical trunk. Selective arteriogram (b) of right thyrocervical trunk showing collateral supply to a bronchial artery branch. No active extravasation demonstrated, small pseudoaneurysm seen.

In the second case, MRI demonstrated the likely site of recent hemorrhage and the abnormal vessel (dilated, tortuous branch arising from the thyrocervical trunk and supplying the bronchial artery) which was subsequently empirically embolized.

\section{Discussion}

Acute gastrointestinal bleeding (AGIB) often produces significant barriers to diagnosis and treatment despite advances in medical technology. The mortality rate ranges from $3.6 \%$ to $21 \%$ [3]-[5], with further increase in high-risk patient populations.

Endoscopy is considered the first line investigation and the gold standard in detection and treatment of AGIB. However, there are limitations to both upper GI endoscopy and colonoscopy which lead to non-diagnostic results in a significant number of cases [6]. Furthermore, endoscopy is unable to localize bleeding from the majority of the small bowel as it is not accessible with the equipment currently available. Capsule endoscopy does offer a potential solution in some tertiary centers but it is time consuming and expensive.

Radiological methods commonly used to diagnose AGIB are nuclear scintigraphy and digital subtraction angiography (DSA). These modalities are often used to complement endoscopic techniques, or may be the primary diagnostic test where endoscopy fails to detect the source of hemorrhage. In more recent years, a number of studies have been published [7], [8] which advocate the use of Multidetector CT (MDCT) as it is generally faster to perform compared with scintigraphy, less invasive and usually easier to obtain than conventional DSA. MDCT has been compared favorably to both DSA and endoscopy and is often used as a guide for subsequent intervention [8]. However MDCT involves use of significant doses of ionizing radiation, especially given the multiphasic protocols suggested in current literature and utilized in most centers [9]. Nuclear scintigraphy also requires using ionizing radiation, often offering poor anatomic localization due to limitations in resolution.

Magnetic Resonance Imaging (MRI) has become a widely available imaging technique. MRI is non invasive and requires neither the use of ionizing radiation nor iodinated contrast and therefore offers an attractive alternative, especially for imaging younger patients and those with allergies to iodinated contrast.

One of the limitations of contrast-enhanced MR angiography lies in the fact that standard extracellular agents are used in nearly all applications. This class of contrast agents is hydrophilic, causing them to diffuse through extracellular fluids rather than bind selectively to a target [10], resulting in a relatively short intravascular half-life. Their use in steady-state vascular imaging is therefore not feasible as they rapidly diffuse into surrounding tissues. However, intravascular contrast agents have the ability to remain within the circulation for long periods, allowing multiple steady-state imaging series to be performed. Theoretically, these agents should be useful in detection of slow hemorrhage.

To date, gadofosveset trisodium (Trade name Ablavar, Lantheus Medical Imaging Inc., North Billerica MA, 
USA) is the first FDA—approved gadolinium-based MR contrast agent designed to image the blood pool [11]. Gadofosveset binds reversibly in a non-covalent manner to human albumin. With this blood pool contrast agent, interpretable steady-state images of blood vessels can be obtained for up to 30 minutes post injection [12]. Furthermore, binding to albumin also enhances relaxivity, up to 6 - 10 times greater than with non-protein bound agents, which results in improved contrast. Steady-state MRA with gadofosveset has been favorably compared with non contrast (Time of Flight) MRA and contrast-enhanced first-pass MRA in a few studies which have been centered mainly on the detection of vascular pathology in various locations rather than the localization of bleeding [13]. Although at least one experimental animal model has been studied [14], to date there have been no large studies in humans designed to investigate the efficacy of blood pool MR contrast agents in the localization of bleeding.

Our cases illustrate the potential utility of steady-state MRA as a first-line imaging modality in localizing occult hemorrhage in clinically stable patients. In the case of lower GI bleeding there was excellent correlation among the three imaging modalities, and although the repeat colonoscopy did not demonstrate active bleeding, it did reveal ascending colonic and cecal ulcers as likely sources of the hemorrhage. In the case of the patient with hemoptysis, MRI demonstrated the site of recent hemorrhage and accurately depicted the culprit vessel. Again there was excellent correlation with the DSA. According to Yoon et al. [15], in over 90\% of hemoptysis cases requiring intervention or surgery, a bronchial artery is responsible for the bleeding. On CT, bronchial arteries that are greater than $2 \mathrm{~mm}$ in diameter are considered abnormal and those which have been demonstrated as sources of hemorrhage have been shown to be larger than those shown not to be bleeding [15]. MRI with steady-state imaging offers an alternate means of assessing the bronchial and pulmonary arterial anatomy.

Although larger studies are required, we believe that steady-state imaging with a blood-pool agent such as Gadofosveset offers an excellent alternative to nuclear scintigraphy and CT in the localization of hemorrhage and guiding subsequent intervention. Blood-pool agents have been investigated, with promising results, for their use in detection of arterial stenosis and endoleak following Endovascular Aneurysm Repair (EVAR) [16].

Disadvantages of using MRI for this purpose may include long acquisition times, limitations due to claustrophobia and greater cost than other modalities. Data must therefore be gathered pertaining to factors such as optimization of contrast dose, patient selection and preparation, minimum detectable rates of hemorrhage and cost effectiveness of these techniques prior to their widespread use in clinical practice.

\section{Conclusion}

Performing contrast enhanced MRI with a blood-pool contrast agent such as gadofosveset to detect and localize hemorrhage poses a number of technical and logistical problems which currently preclude its widespread use. However, it may provide a valuable alternative in special circumstances such as in patients with iodine allergies and in cases where multiple scans are required and the cumulative dose of ionizing radiation may become onerus.

More data is required regarding safety, cost-effectiveness, sensitivity and efficiency of the technique prior to its introduction into the average imaging department.

\section{References}

[1] Potter, G.D. and Sellin, J.H. (1988) Lower Gastrointestinal Bleeding. Gastroenterology Clinics of North America, 17, 341-355.

[2] Moody, A.R. (2003) Magnetic Resonance Direct Thrombus Imaging. Journal of Thrombosis and Haemostasis, 1, 1403-1409. http://dx.doi.org/10.1046/j.1538-7836.2003.00333.x

[3] Jaeckle, T., Stuber, G. and Hoffman, M.H. (2008) Acute Gastrointestinal Bleeding: Value of MDCT. Abdominal Imaging, 33, 285-293. http://dx.doi.org/10.1007/s00261-007-9263-8

[4] Al Qahtani, A.R., Satin, R., Stern, J., et al. (2002) Investigative Modalities for Massive Lower Gastrointestinal Bleeding. World Journal of Surgery, 26, 620-625. http://dx.doi.org/10.1007/s00268-001-0279-X

[5] Walsh, R.M., Anain, P. and Geisinger, M. (1999) Role of Angiography and Embolization for Massive Gastroduodenal Hemorrhage. Journal of Gastrointestinal Surgery, 3, 61-65. http://dx.doi.org/10.1016/S1091-255X(99)80010-9

[6] Vreeburg, E.M., Snel, P. and De Brujine, J.W. (1997) Acute Upper Gastrointestinal Bleeding in the Amsterdam Area: Incidence, Diagnosis and Clinical Outcome. American Journal of Gastroenterology, 92, 236-243.

[7] Ernst, O., Bulois, P., Sain-Drenant, S., Leroy, C., Paris, J.-C. and Sergent, G. (2003) Helical CT in Acute Lower Ga- 
strointestinal Bleeding. European Radiology, 13, 114-117.

[8] Miller, F.H. and Hwang, C.M. (2004) An Initial Experience Using Helical CT Imaging to Detect Obscure Gastrointestinal Bleeding. Journal of Clinical Imaging, 28, 245-251. http://dx.doi.org/10.1016/S0899-7071(03)00193-1

[9] Frattaroli, F., Casciani, E., Spoletini, D., et al. (2009) Prospective Study Comparing Multi-Detector Row CT and Endoscopy in Acute Gastrointestinal Bleeding. World Journal of Surgery, 33, 2209-2217. http://dx.doi.org/10.1007/s00268-009-0156-6

[10] Kotek, J., Lebduskova, P., Hermann, P., et al, (2003) Lanthanide (III) Complexes of Novel Mixed Carboxylic-Phosphorus Acid Derivatives of Diethylenetriamine: A Step towards More Efficient MRI Contrast Agents. Chemistry, 9, 5899-5915. http://dx.doi.org/10.1002/chem.200305155

[11] Henness, S. and Keating, G. (2006) Gadofosveset. Drugs, 66, 851-857. http://dx.doi.org/10.2165/00003495-200666060-00008

[12] Fink, C., Goyen, M. and Lotz, J. (2007) Magnetic Resonance Angiography with Blood-Pool Contrast Agents: Future Applications. European Radiology, 17, B38-B44.

[13] Anzidei, M., Napoli, A. and Marincola, B. (2009) Gadofesveset-Enhanced MR Angiography of Carotid Arteries: Does Steady-State Imaging Improve Accuracy of First-Pass Imaging? Comparison with Selective Digital Subtraction Angiography. Radiology, 251, 457-466. http://dx.doi.org/10.1148/radiol.2512081197

[14] Hilfiker, P.R., Weishaupt, D. and Kacl, G.M. (1999) Comparison of Three Dimensional Magnetic Resonance Imaging in Conjunction with a Blood Pool Contrast Agent and Nuclear Scintigraphy for the Detection of Experimentally Induced Gastrointestinal Bleeding. Gut, 45, 581-587. http://dx.doi.org/10.1136/gut.45.4.581

[15] Yoon, W., Kim, J.K., Kim, Y.H., et al. (2002) Bronchial and Nonbronchial Systemic Arterial Embolization for Life-Threatening Hemoptysis: A Comprehensive Review. Radiographics, 22, 1395-1409. http://dx.doi.org/10.1148/rg.226015180

[16] Cornelissen, S., Prokop, M. and Verhagen, H. (2010) Detection of Occult Endoleaks after Endovascular Treatment of Abdominal Aortic Aneurysm Using Magnetic Resonance Imaging with a Blood Pool Agent. Investigative Radiology, 45, 1-6. http://dx.doi.org/10.1097/RLI.0b013e3181e992ac 